\title{
Relationship between Helicobacter pylori and
}

\section{expression of programmed death-I and its ligand in gastric intraepithelial neoplasia and early-stage gastric cancer}

This article was published in the following Dove Press journal:

Cancer Management and Research

\author{
Bo Shen' \\ Aihua Qian' \\ Wenji Lao' \\ Weiguang $\mathrm{Li}^{\prime}$ \\ Xi Chen' \\ Benyan Zhang ${ }^{2}$ \\ Huafeng Wang ${ }^{2}$ \\ Fei Yuan ${ }^{2}$ \\ Yunwei Sun' \\ 'Department of Gastroenterology of \\ Ruijin Hospital, Shanghai Jiao Tong \\ University School of Medicine, Shanghai \\ 200025, People's Republic of China; \\ ${ }^{2}$ Department of Pathology of Ruijin \\ Hospital, Shanghai Jiao Tong University \\ School of Medicine, Shanghai 200025, \\ People's Republic of China
}

Correspondence: Fei Yuan Department of Pathology of Ruijin Hospital, Shanghai Jiao Tong University School of Medicine, Shanghai, 200025, People's Republic of China daphny20I4@I63.com

Yunwei Sun

Department of Gastroenterology of Ruijin Hospital, Shanghai Jiao Tong University School of Medicine, Shanghai 200025, People's Republic of China Email sun_yunwei@qq.com
Background: Many studies have shown that programmed cell death protein 1 (PD-1) and its ligand, PD-L1, are expressed in advanced gastric cancer. Furthermore, detection of these proteins is associated with infiltrating CD8+ T-cells, indicating that an adaptive immune resistance mechanism occurs in advanced gastric cancer. However, PD-L1 and PD-1 expression in gastric intraepithelial neoplasia and early-stage gastric cancer (EGC) has yet to be elucidated.

Patients and methods: Fifty-four resections of low-grade intraepithelial neoplasia (LGIN), high-grade intraepithelial neoplasia (HGIN), and EGC were stained by immunohistochemistry for PD-1, PD-L1, and CD8. CD8+ T-cell densities both within tumors and in the tumor-stromal interface were analyzed. Flow cytometry (FACS) was used to analyze the PD1 expression in tumor tissues and peripheral blood mononuclear cells. Furthermore, the relationship between Helicobacter pylori $(\mathrm{Hp})$ infection and PD-1 and PD-L1 was also evaluated

Results: We demonstrated that PD-L1 expression was significantly increased in HGIN and EGC compared with LGIN, and both PD-1 and PD-L1 showed similar expression patterns, being mainly detected in infiltrating immune cells. FACS also showed that PD-1 was expressed on both CD4+ and CD8+ T-cells. However, no difference was found in CD8+ T-cell infiltration between LGIN and HGIN+EGC, and this was not not found to be associated with PD-L1 or PD-1 expression. However, Hp infection was significantly associated with expression of PD-L1 and PD-1.

Conclusions: The PD-1/PD-L1 checkpoint is involved in intraepithelial neoplasia and EGC, but an adaptive immune resistance mechanism does not occur. Expression of PD-1/ PD-L1 is also associated with $\mathrm{Hp}$ infection, and so Hp infection may be an important initiating factor.

Clinical Trial Registration information: This study was approved by the Institutional Review Board of Ruijin Hospital and written informed consent was obtained from all patients. Keywords: PD-L1, PD-1, Helicobacter pylori, LGIN, HGIN

\section{Introduction}

Gastric cancer ranks among the top five cancers, with highest mortality rates with a 5-year survival rate of around $30 \%{ }^{1}$ In recent years, checkpoint inhibitors have been achieving encouraging results in gastric cancer, including inhibitors of programmed death-1 (PD-1) and its ligand PD-L1. ${ }^{2-4}$ PD-1 is transiently expressed on 
the surface of activated T-cells and other immune cells including $\mathrm{CD} 8+\mathrm{T}, \mathrm{CD} 4+\mathrm{T}$, natural killer (NK) $\mathrm{T}$ - and B-cells, activated monocytes and dendritic cells. ${ }^{5}$ Binding to its cognate ligand PD-L1, which is constitutively expressed on many cell types, induces apoptosis in the PD-1-expressing cell. Thus, the immune response can be limited effectively. ${ }^{6}$ PD-1 and PD-L1 together can limit T-cell activity and act as a T-cell coinhibitory pathway to balance costimulatory signals. This is essential for maintaining peripheral tolerance and preventing excessive tissue damage in the process of clearing infection. ${ }^{7}$ However, in the tumor microenvironment, PD-L1 can become upregulated on the tumor cell, resulting in reduction of the anti-tumor immune response and evasion of T-cellmediated cytotoxicity.

In 2017, a PD-1 inhibitor Pembrolizumab was approved for the treatment of patients with localized advanced or metastatic PD-L1-positive gastric cancer, ${ }^{2,8}$ with immunohistochemistry of PD-L1 remaining an important standard to guide the application of such PD-1 inhibitors. ${ }^{8-10}$ Our previous results have shown that PD-L1 is not only expressed in tumor cells, but also in immune cells. The positivity rate of PD-L1 in tumor cells was $10-35 \%$ and $42-75 \%$ in immune cells. with an obvious correlation between PD-1 and PD-L1. ${ }^{9,11}$ CD8+ T-cells were distinctly localized at the invasive tumor margin and facilitate the PD-1/PD-L1 immune inhibitory axis so, therefore, may predict response to therapy. ${ }^{5,12}$ This relationship has been proven in advanced gastric cancer, which suggests that an adaptive immune resistance-type mechanism occurs in advanced gastric cancer. ${ }^{9,10}$

However, whether PD-1 and PD-L1 are expressed in gastric intraepithelial neoplasia and early-stage gastric cancer is unknown. Intraepithelial neoplasia is the initial stage of carcinoma development, and PD-1/PD-L1 expression has been reported in intraepithelial neoplasia, including cervical intraepithelial neoplasia (CIN) and sessile serrated adenoma. ${ }^{13-15}$ In CIN, PD-L1 expression is higher in patients with human papilloma virus (HPV) infection than those without infection, with HPV-negative patients showing almost negligible PD-1 expression, thus suggesting that HPV infection may induce PD-L1/PD-1 expression. ${ }^{15}$

During chronic infection, PD-L1/PD-1 expression is upregulated and converts effector T-cells into exhausted T-cells. Therefore, chronic infection is also an important factor in the upregulation of PD-L1/PD-1 expression. Helicobacter pylori (Hp) is an important pathogen that chronically infects the gastric tract, causing inflammation, ulcers, and cancer. ${ }^{16} \mathrm{Hp}$ infection is considered to be a major risk factor for gastric cancer, and is classified as a class 1 carcinogen by the World Health Organization. ${ }^{17}$ Over $90 \%$ of gastric adenocarcinomas are the result of long-term mucosal inflammation; ${ }^{18}$ atrophic gastritis progresses to intestinal metaplasia, followed by dysplasia and carcinoma. ${ }^{18}$ Studies have shown that eradication of $\mathrm{Hp}$ can reduce the risk of developing gastric cancer and abolish the inflammatory response, while early treatment prevents progression to preneoplastic lesions. ${ }^{19}$ In the absence of intestinal metaplasia, the gastric atrophy can even be reversed by Hp eradication. ${ }^{19}$

PD-L1 expression on gastric epithelial cells was significantly induced following $\mathrm{Hp}$ infection in vitro. ${ }^{20}$ Furthermore, Hp-positive human gastric biopsies showed higher PD-L1 expression compared with Hp-negative gastric biopsies. ${ }^{21}$ The relationship between Hp and PD-1/PDL1 expression in low-grade intraepithelial neoplasia (LGIN), high-grade intraepithelial neoplasia (HGIN), and early gastric cancer (EGC) is clearly worth investigating. Here we investigated PD-1 and PD-L1 expression in gastric intraepithelial neoplasia and EGC to determine whether this checkpoint occurs in the early stages of gastric carcinogenesis. We also examined CD8+ T-cells at the invasive tumor interface and the tumor microenvironment, and the relationship between PD-L1 and CD8+ T-cells in gastric cancers. Finally, we determined the relationship between PD-L1 expression and Hp.

\section{Materials and methods Study population and design}

A total of 54 patients with LGIN (low grade intraepithelial neoplasia), HGIN (high grade intraepithelial neoplasia), and EGC (early gastric cancer) and at least one measurable lesion, treated with ESD (endoscopic submucosal dissection) between January 2015 and December 2017. There were $21 \mathrm{LGIN}, 11 \mathrm{HGIN}$, and 22 EGC patients in our research.

All diagnoses were detected by gastroscope white light (EG-590 ZW, Fujifilm), Linked Color Imaging (EG-590 $\mathrm{ZW}$, Fujifilm), and Blue laser imaging (BLI) (EG-590 ZW, Fujifilm), finally confirmed by ESD of the primary tumor. All specimens were diagnosed with the stage of tumor (LGIN, HGIN and EGC) and the depth of tumor invasion by two professional pathologists.

This study was approved by the Institutional Review Board of Ruijin Hospital, and written informed consent was obtained from all patients. 


\section{Assessment of Hp positivity}

All patients had detected Hp infection by immunohistochemical method before ESD treatment. Immunohistochemical staining was performed to identify HP-positive bacteria in the gastric pits or glandular lumens with scattered or clustered curved short rod-shaped, comma-shaped, S-shaped, or $\mathrm{C}$-shaped bacteria under high power microscopy.

\section{Immunohistochemistry and assessment of PD-LI, PD-I, and CD8 expression}

The primary LGIN, HGIN, and gastric tumors was sectioned $(5 \mu \mathrm{m}$ thick) and routinely stained with H\&E. Immunostaining was performed manually using a rabbit monoclonal anti-PD-L1 antibody (1:50, SP142, Roche), anti-PD-1 antibody (1:50, NAT105, Abcam), and anti-CD 8 antibody (1:100, ab4055, Abcam). For the evaluation of the PD-L1 expression in tumor cells, only the membranous staining and the following immunoreactivity scoring system (IRS) were evaluated. ${ }^{10}$ For the evaluation of the PDL1 expression, including tumor infiltrating lymphocytes (TILs) and tumor-associated macrophages (TAMs), only the percentage of positive cells was considered, and cases were considered as negative $(<1 \%)$ or positive $(\geq 1 \%) .{ }^{10}$ The percentage of stained cells was evaluated separately by two pathologists. The immunostaining of PD-1 in immune cells was rated as present or absent. ${ }^{10}$ Slides stained for CD8 were annotated for areas of tumor and tumor-stromal interface. CD8+ density was quantified at three representational areas, calculating the average density $/ \mathrm{mm}^{2}$ of the three areas.

\section{Preparation of peripheral blood mononuclear cells (PBMCs)}

Approximately $5 \mathrm{ml}$ of peripheral blood was drawn from the patients on the day before surgery. A Ficoll-Paque (Pharmacia, Uppsala, Sweden) density gradient was used to centrifuge blood samples.

\section{Preparation of gastric tissue dissociation}

Prepare digestion media ( $1 \mathrm{ml}$ for 1 sample): $5 \mu$ DNAse + Collagenase IV in serum-free HBSS/RPMI media. Harvest tissue and place in a small volume of serum-free HBSS/ RPMI. Add PBS to the tissue sample in a petri dish to wash the tissue. Pipette $1 \mathrm{ml}$ digestion medium and cut the tissue using a scissor into fine pieces. Transfer tissue to 12 -well or 6-well plates. Incubate it in the incubator at $37^{\circ} \mathrm{C}$ for 50 minutes $\sim 1$ hour. Place a $100 \mu \mathrm{M}$ filter on a $50 \mathrm{ml}$ falcon tube and filter the single cell suspension through the $100 \mu \mathrm{M}$ mesh. Re-suspend the cells in $2-20 \mathrm{ml}$ PBS (depends on the size of cell pellet); homogenize with the help of pipette mix. Pending for FACS staining.

\section{Flow cytometric analysis}

PBMC and gastric tissue cell suspension were stained for various surface markers: Live/dead-PE-CF594 (BD Biosciences, San Jose, CA, USA), CD45-AF700 (Invitrogen, Grand Island, NY, USA), CD3-Percpcy5.5 (eBiosciences, San Diego, CA, USA), CD4-FITC (eBiosciences), CD8a-APC-eF780 (eBiosciences), and PD-1/isotype-APC (BD Biosciences). Samples were analyzed with a BD LSRFortessa X-20.

\section{Statistical analysis}

The Cochran-Armitage test for trend was used to determine if the probability of positive PD-1 and PD-L1 expression increased with increasing levels of CD8 expression. The correlation between the depth of invasion and the rate of PD-L1 and PD-1 positive were analyzed using the Spearman test. Other associations were assessed with $\chi^{2}$ or Fisher's exact tests as appropriate. Means were compared with two-sample Student's $t$-test. Statistical analyses were performed using SPSS (version 21; SPSS Inc, Chicago, IL, USA), and results were considered statistically significant at $* P<0.05 ; * * P<0.01$; and $* * * P<0.001$. The Cochran-Armitage test for trend were performed using SAS V.9.2 (SAS Institute, Cary, NC, USA).

\section{Results}

\section{Patient characteristics}

Fifty-four patients undergoing endoscopic submucosal dissection were included in this study, including 21 LGIN, 11 HGIN, and 22 EGC. Basic characteristics of all patients are shown in Table 1. Representative white light, live cell imaging, bioluminescence imaging, and hematoxylin and eosin histological sections of LGIN, HGIN, and EGC are shown in Figure 1. HGIN and EGC were combined into a single group (HGIN+EGC). The mean ages of patients with LGIN and HGIN+EGC were $65.2 \pm 1.8$ and $64.8 \pm 1.2$ years, respectively. There were 29 female and 25 male patients included in this study, with 10 and 15 males in the LGIN and HGIN+EGC groups, respectively. Of the LGIN group, 14 patients had tumors located in the antrum, and seven were located in the body of the stomach. Of the 
Table I Patient's characteristics

\begin{tabular}{|l|l|l|l|}
\hline & LGIN & HGIN+EGC & $P$ \\
\hline $\begin{array}{l}\text { Age (years) } \\
\text { Median (range) }\end{array}$ & $65.2(51-80)$ & $64.8(52-77)$ & \\
$\begin{array}{l}\text { Male } \\
\text { Female }\end{array}$ & $10(47.6 \%)$ & $15(45.5 \%)$ & 0.858 \\
$\begin{array}{l}\text { Tumor location } \\
\text { Antrum }\end{array}$ & $11(53.4 \%)$ & $18(54.5 \%)$ & \\
$\begin{array}{l}\text { Body } \\
\text { Hp infection }\end{array}$ & $14(66.7 \%)$ & $19(57.6 \%)$ & 0.504 \\
$\begin{array}{l}\text { Positive } \\
\text { Negative }\end{array}$ & $7(33.3 \%)$ & $14(42.4 \%)$ & \\
Neutrophil (Mean, SD) & $2.8 \pm 0.18 * 10^{9}$ & $3.5 \pm 0.26 * 10^{9}$ & 0.0383 \\
\hline
\end{tabular}

Abbreviations: $\mathrm{EGC}$, early gastric cancer; $\mathrm{HGIN}$, high grade intraepithelial neoplasia; $H_{p}$, Helicobacter pylori; LGIN, low grade intraepithelial neoplasia.

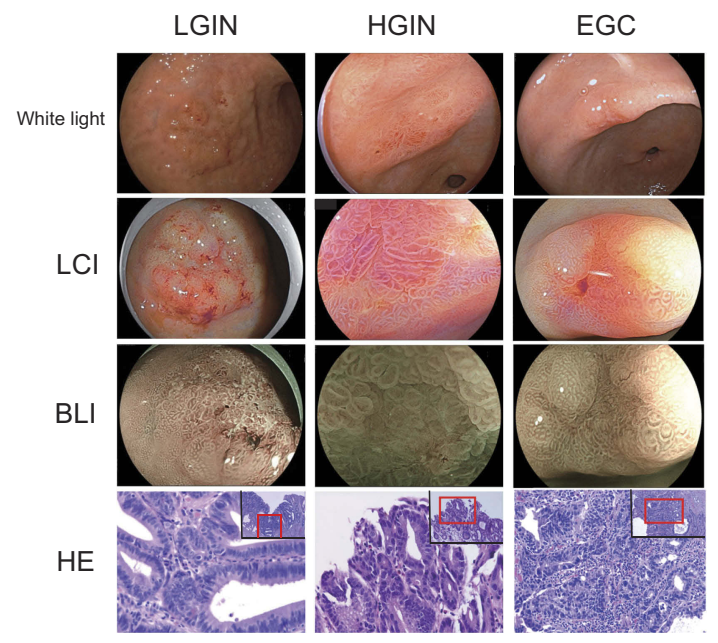

Figure I The representative white light, $\mathrm{LCl}, \mathrm{BLI}$ and $\mathrm{H \& E}$ histological sections of LGIN, HGIN, and EGC.

Abbreviations: BLI, Blue Laser Imaging; EGC, early-stage gastric cancer; HGIN, High-grade Intraepithelial neoplasia, LCI, Linked Color Imaging; LGIN, Low-grade Intraepithelial neoplasia.

HGIN and EGC patients, 19 had tumors located in the antrum and 14 in the body (Table 1).

\section{Expression of PD-LI in LGIN, HGIN, and EGC}

Of the 54 patients investigated, 24 (44.4\%) were PD-L1positive and $30(55.6 \%)$ were negative. (Figures $2 \mathrm{~A}-\mathrm{H})$, but the PD-L1 expression between LGIN ( $n=21)$ and HGIN + EGC $(\mathrm{n}=33)$ groups was significantly different $(P=0.003$; Figure 2I). In the LGIN group, four (19.0\%) were positive and $17(81.0 \%)$ were negative; however, in the HGIN+EGC group, $20(60.6 \%)$ were positive and $13(39.4 \%)$ were negative. PD-L1 expression was mainly located in the immune cells, including tumor infiltrating lymphocytes and tumorassociated macrophages, rather than in the tumor cells, which is different to the expression pattern seen in advanced cancer. We further subdivided HGIN+EGC groups into gastric carcinomas limited to the epithelial layer $(n=11)$ and those with a depth of invasion into the muscularis mucosa $(n=16)$ and submucosa $(n=6)$. In the gastric carcinomas limited to the epithelial layer, six $(54.5 \%)$ were PD-L1 positive and five (45.5\%) were PD-L1 negative. In gastric carcinomas with depth of invasion into the muscularis mucosa, $10(62.5 \%)$ were PD-L1 positive and six (37.5\%) were PD-L1 negative, while for gastric carcinomas with depth of invasion into the submucosa, four $(66.7 \%)$ were PD-L1 positive and two $(33.3 \%)$ were PD-L1 negative. The differences between groups did not achieve statistical significance $(P=0.901)$, indicating that PD-L1 expression was not correlated with the depth of invasion (Figure 2J).

\section{Expression of PD-I in LGIN, HGIN, and EGC}

Of our 54 patients, 24 (44.4\%) were PD-1 positive and 30 (55.6\%) were negative (Figure $3 \mathrm{~A}-\mathrm{H}$ ). The expression of PD-1 was different between the dysplasia and EGC groups. In LGIN patients, five (23.8\%) were PD-1 positive and $16(76.2 \%)$ were negative, While of the HGIN+EGC group, $18(45.5 \%)$ were positive and $15(54.5 \%)$ were negative. Thus, PD-1 expression significantly correlated with tumor stage $(P=0.026$; Figure $3 \mathrm{I})$. In the gastric carcinomas limited to the mucosa, five $(45.5 \%)$ were PD1 positive and six (54.5\%) were PD-1 negative. In gastric carcinomas with depth of invasion into the muscularis mucosa, eight $(50.0 \%)$ were PD-1 positive and eight $(50.0 \%)$ were PD-1 negative, while in gastric carcinomas with depth of invasion into the submucosa, five (83.3\%) were PD-1 positive and one (16.7\%) were negative. Similar to PD-L1, PD-1 expression showed no correlation with the depth of invasion. ( $P=0.206$; Figure $3 \mathrm{~J})$.

\section{The flow cytometry analysis of PD-I expression on CD4+ and CD8+ $T$ cells in LGIN, HGIN and EGC}

Four patients with HGIN+EGC and two healthy people were analyzed by flow cytometry to assess expression of PD-1 on CD4+ and CD8+ T-cells. Peripheral blood mononuclear cells (PBMCs) from both healthy and HGIN+ EGC individuals were negative for PD-1 on CD4+ and CD8+ T-cells. However, in gastric cancer tissues of 

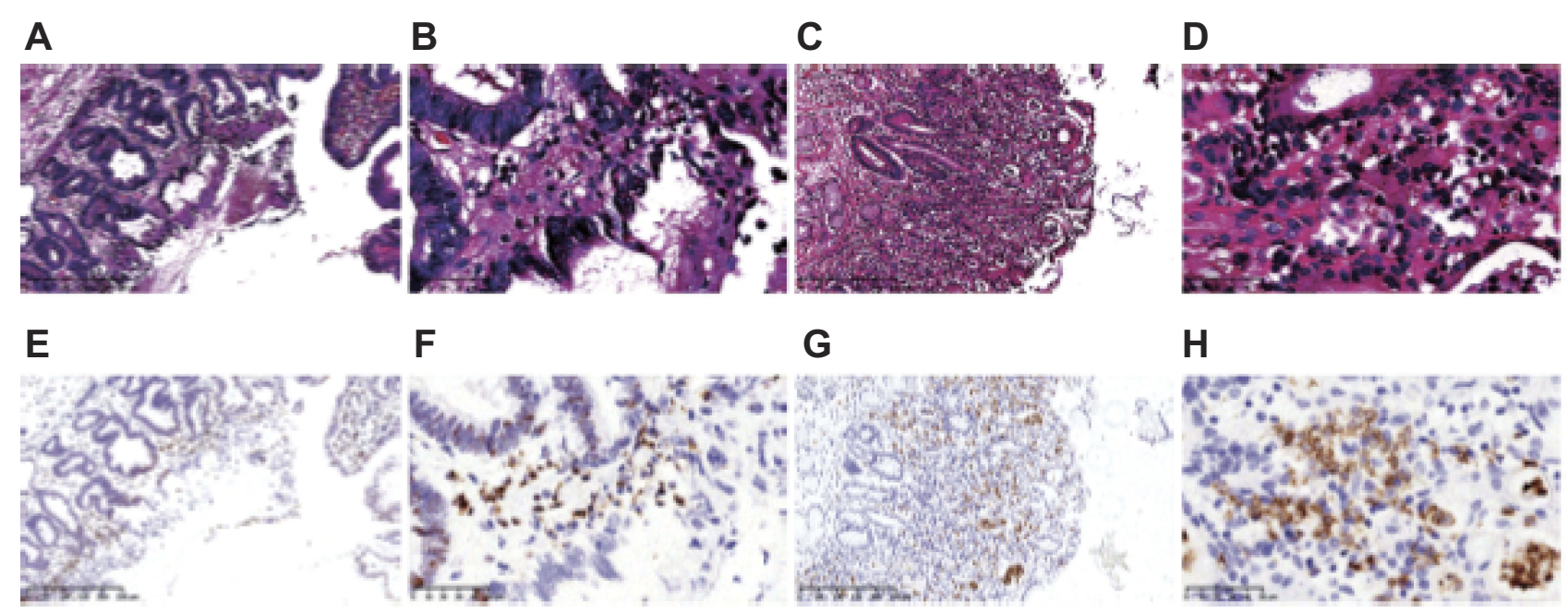

$\mathbf{F}$

G
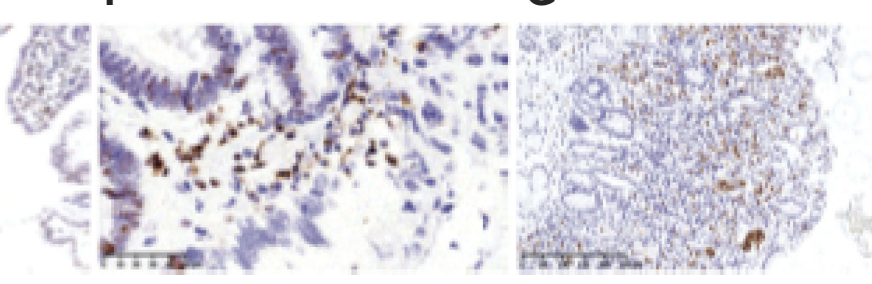

H

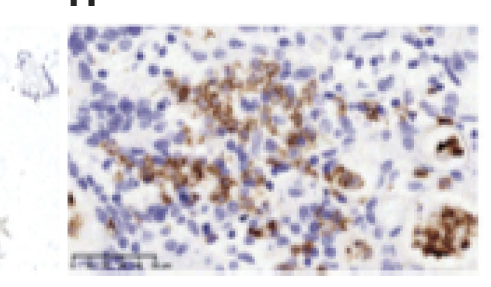

I

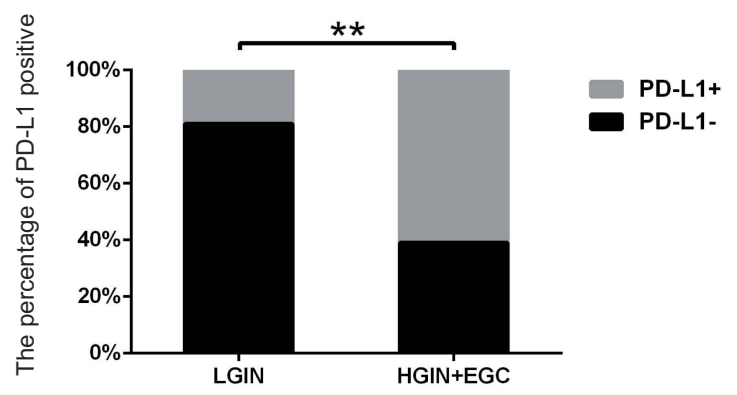

J

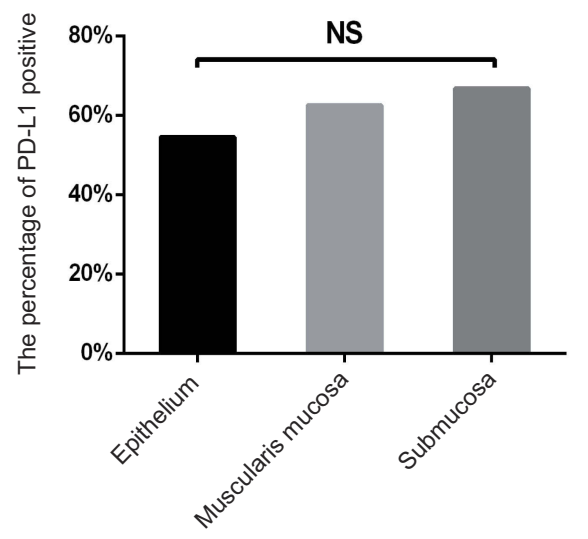

Figure 2 The expression of PD-LI and its association with stage in LGIN, HGIN, and EGC. (A-H) The representative expression of PD-LI in LGIN (A, B, E, F), HGIN+EGC (C, D, G, H); shown at $\times 100(\mathbf{A}, \mathbf{C}, \mathbf{E}, \mathbf{G})$ and $\times 400(\mathbf{B}, \mathbf{D}, \mathbf{F}, \mathbf{H})$ original magnification; (I) The correlation between stage and PD-LI expression, which was determined using $\chi^{2}$ test; (J) The correlation between the depth of invasion and PD-LI expression, which were also determined using the Spearman's rank correlation coefficient test.

Note: $* * P<0.01$.

Abbreviations: EGC, early-stage gastric cancer; HGIN, High-grade Intraepithelial neoplasia; LGIN, Low-grade Intraepithelial neoplasia; NS, not statistically significant; PD$\mathrm{LI}$, programmed death-ligand I.

patients with HGIN+EGC, one patient demonstrated PD-1 positivity in both $\mathrm{CD} 4+$ and $\mathrm{CD} 8+\mathrm{T}$-cell populations (Figure 4B). CD4+ T-cells were PD-1-positive, while CD8+ T-cells were negative in two patients, and, in the remaining patient, both $\mathrm{CD} 4+\mathrm{T}$-cell and $\mathrm{CD} 8+\mathrm{T}$-cell were PD-1 negative. In normal gastric mucosa, PD-1 on CD4+ and CD8+ T-cells were negative (Figure 4A).

\section{CD8 expression in LGIN, HGIN, and EGC}

As facilitators of adaptive immune resistance, CD8+ T-cells are associated with PD-L1 expression in gastric cancer. We measured the density of CD8+ T-cells in representative intratumoral and tumor-stromal interface sections from each patient, and compared the density of
CD8+ T-cells between the two sites in LGIN and HGIN + EGC (Figure 5A). The intratumoral density of CD8+ T-cells in the 21 LGIN patients was $142.9 \pm 17.94 / \mathrm{mm}^{2}$, while HGIN+EGC patients showed a density of 148.1 $\pm 21.44 / \mathrm{mm}^{2}$. The density of CD8 + T-cells at the tumorstromal interface was $211.6 \pm 34.01 / \mathrm{mm}^{2}$ and 250.6 $\pm 32.40 / \mathrm{mm}^{2}$ in LGIN and HGIN+EGC, respectively. There was no significant difference between LGIN and HGIN+EGC in either intratumoral or tumor-stromal interface sites $(P=0.8685$ and $P=0.4291$, respectively; Figure 5B). However, the density of CD8+ T-cells in the tumor-stromal interface was 1.5-fold higher than that observed intratumorally $\left(224.9 / \mathrm{mm}^{2}\right.$ vs $149.2 / \mathrm{mm}^{2}$; $P=0.0005$; Figure 5C). 

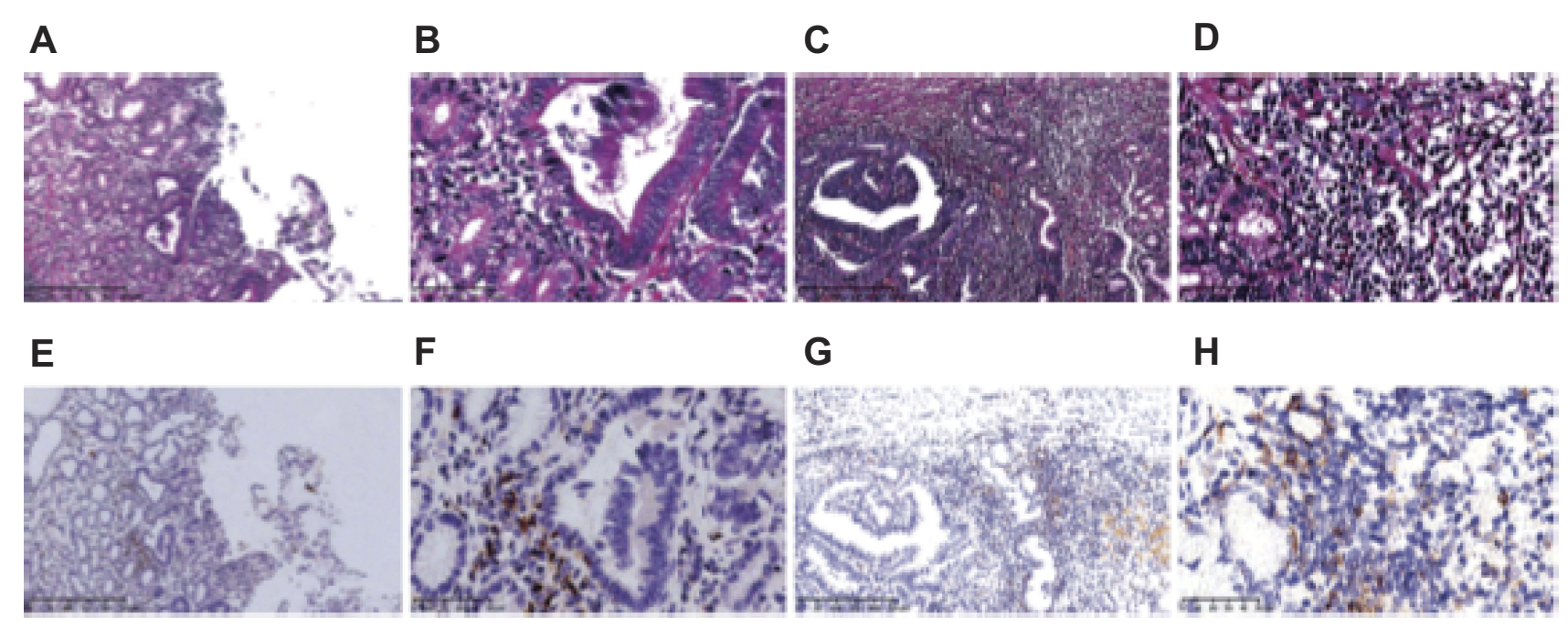

$\mathbf{F}$

\section{G}

$\mathrm{H}$
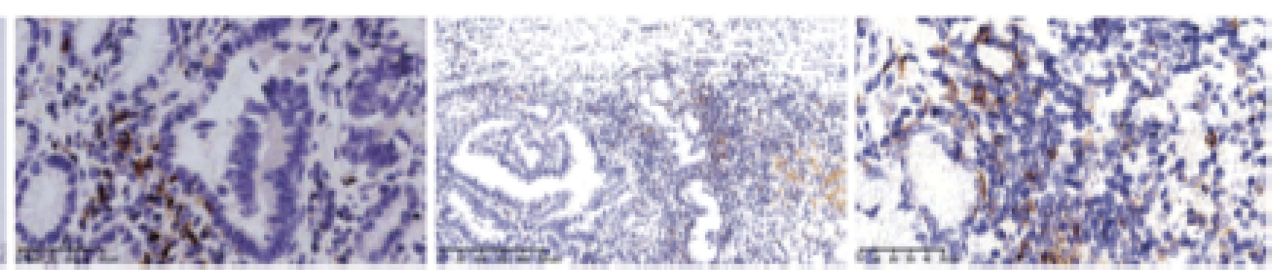

I
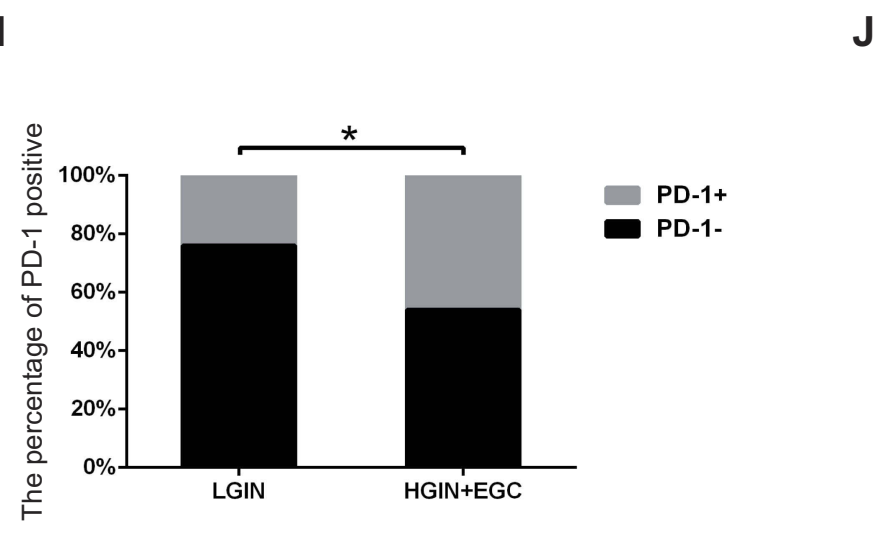

J

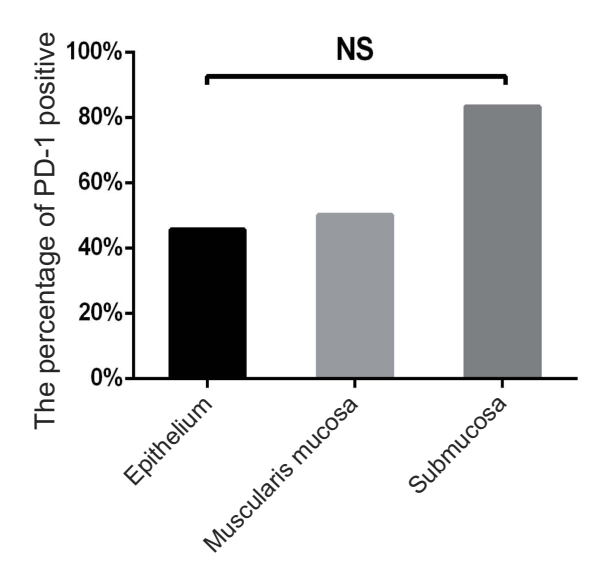

Figure 3 The expression of PD-I and its association with stage in LGIN, HGIN, and EGC. (A-H) Representative expression of PD-I in LGIN (A, B, E, F), HGIN+EGC (C, D, G, H), shown at $\times 100(\mathbf{A}, \mathbf{C}, \mathbf{E}, \mathbf{G})$ and $\times 400(\mathbf{B}, \mathbf{D}, \mathbf{F}, \mathbf{H})$ original magnification; (I) The correlation between stage and PD-I expression, which were determined using $\chi^{2}$ test; $(\mathbf{J})$ The correlation between the depth of invasion and PD-I expression, which were also determined using the Spearman's rank correlation coefficient test. Note: $* P<0.05$.

Abbreviations: EGC, early-stage gastric cancer; HGIN, High-grade Intraepithelial neoplasia; LGIN, Low-grade Intraepithelial neoplasia; NS, not statistically significant; PD-I, programmed death-I.

\section{Relationship between CD8+ T-cell density and PD-LI/PD-I expression in LGIN, HGIN, and EGC}

We compared average CD8+ T-cell densities in LGIN and HGIN+EGC with or without expression of PD-L1/PD-1. Intratumoral and stromal CD8+ T-cell densities were stratified by quartiles: low $\left(<147 / \mathrm{mm}^{2}\right)$, mid $\left(147-500 / \mathrm{mm}^{2}\right)$ and high $\left(>500 / \mathrm{mm}^{2}\right)$ (9).(9) The correlation between CD8 + density and PD-1/PD-L1 expression was then determined. In the low CD8+ quartile, $41.2 \%$ of tumors were PD-L1 positive, while $44.4 \%$ of tumors with mid CD8 density and $50 \%$ of tumors with high CD8 density were PD-L1 positive $(P=0.8115)$. While $50.0 \%$ and $37.1 \%$ of tumors with low or mid stromal CD8 densities, respectively, were PD-L1 positive, $66.7 \%$ of tumors with high stromal CD8 densities were PD-L1 positive. Thus, neither PD-1 nor PD-L1 was correlated with the density of CD8+ T-cells in either LGIN or HGIN+EGC (Figures 5D and E) at intratumoral or stromal interface sites.

\section{Relationship between $\mathrm{Hp}$ infection and expression of PD-I and PD-LI in LGIN, HGIN, and EGC}

Because $\mathrm{Hp}$ is the strongest known risk factor for gastric cancer and is associated with inflammatory immunity, ${ }^{17}$ we wanted to assess whether Hp infection is associated 
A

\section{Normal gastric mucosa}

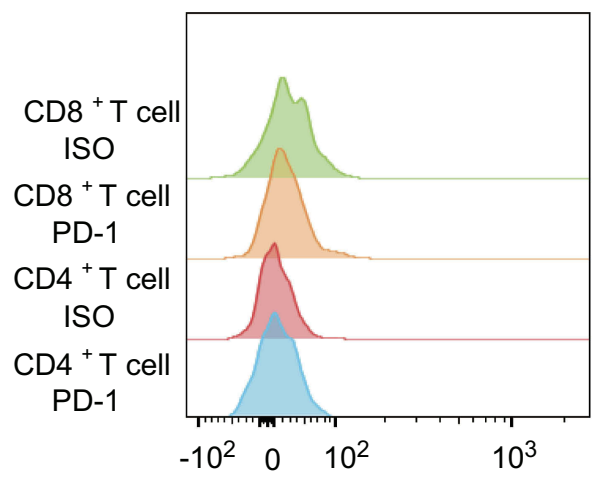

B
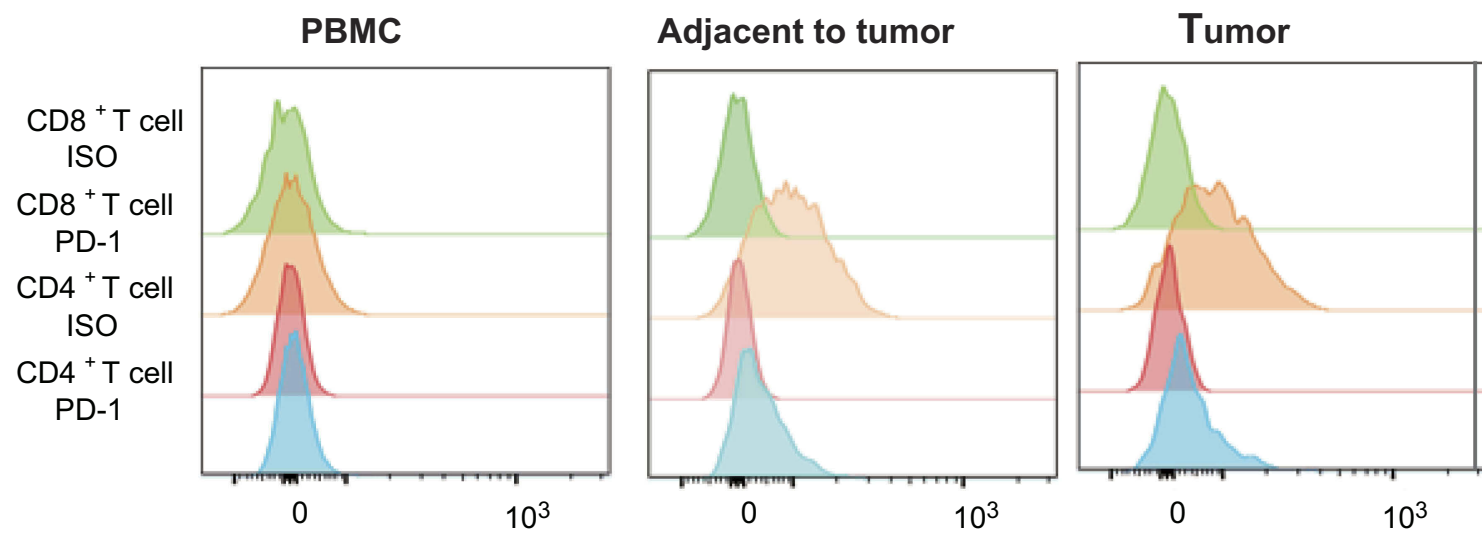

Figure 4 Representative flow expression of PD-I on CD4+ T-cell and CD8+ T-cell in the normal gastric mucosa and in HGIN. (A) The representative flow expression of PD-I and its isotype on CD4+ T-cell and CD8+ T-cell in normal gastric mucosa; (B) The representative flow expression of PD-I and its isotype on CD4+ T-cell and CD8+ T-cell in HGIN.

Abbreviations: HGIN, High-grade Intraepithelial neoplasia; PD-I, programmed death-I.

with tumor immunity. Of our 54 patients with LGIN, HGIN, and EGC, 12 were both PD-L1 and Hp positive, 12 were PD-L1 positive and Hp negative, seven were PDL1 negative and Hp positive, and 23 were negative for both PD-L1 and Hp. Hp was significantly associated with PD-L1 expression ( $P=0.041$; Figure 6A). Similarly, Hp was also associated with $\mathrm{PD}-1$ expression $(P=0.009$; Figure 6B).

\section{Discussion}

In this study, we evaluated the clinical relevance of PDL1 (SP142) and PD-1 (NAT105) expression, and the relationship with $\mathrm{Hp}$ infection in LGIN, HGIN, and EGC. To our knowledge, this study is the first study to provide information on the clinicopathological features of PD-L1 and PD-1 expression in LGIN, HGIN, and early gastric cancer (EGC), including their association with Hp infection.
Many studies have found that PD-L1 and PD-1 are expressed in advanced gastric cancer and are associated with the density of CD8+ T-cells, indicating an adaptive immune resistance mechanism in gastric cancer. ${ }^{9,10}$ PD-L1 expression is mainly located on tumor and immune cells ${ }^{22-24}$ and is correlated with PD-1 expression. ${ }^{10,11}$ In our study, expression of PD-L1 and PD-1 were significantly increased in HGIN and EGC, compared with LGIN. We also found that expression of PD-L1 was mainly localized to infiltrating immune cells, but PD-L1 and PD-1 expression were not associated with CD8+ T-cells infiltrating into the tumors and stroma. These results suggest that the PD-1/PD-L1 inhibitory axis is active at the onset of development of gastric cancer (including LGIN, HGIN, and EGC), and is positively correlated with gastric cancer procession. The results of flow cytometry also proved that PD-1 is active at the onset of development of gastric cancer. However, patients with higher CD8+ T-cell densities did not have higher PD-L1 expression, 
A

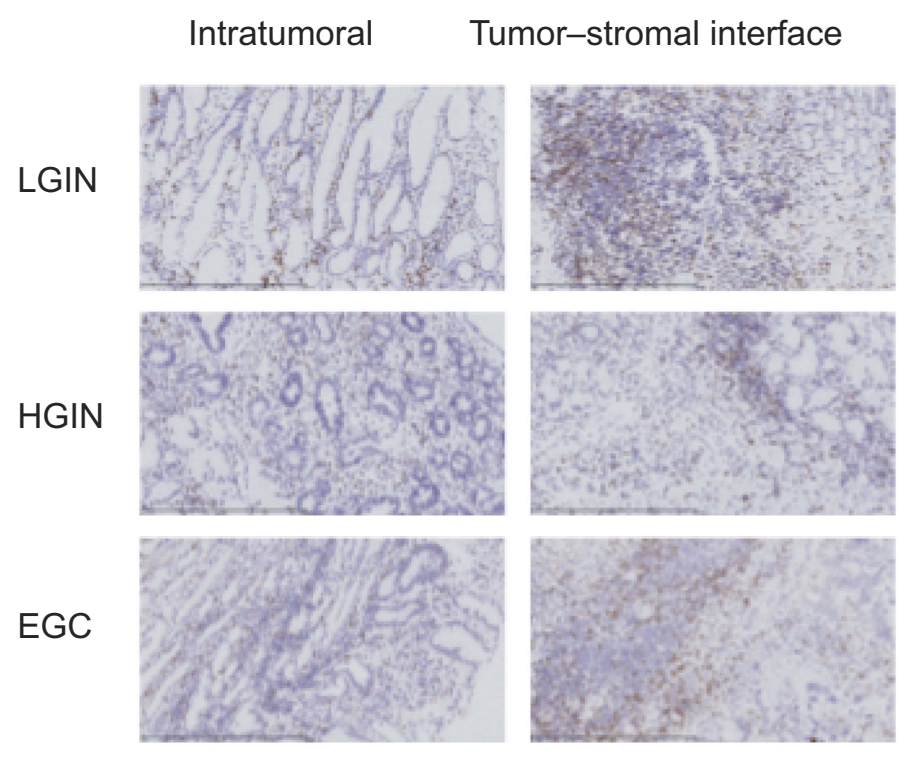

D
B

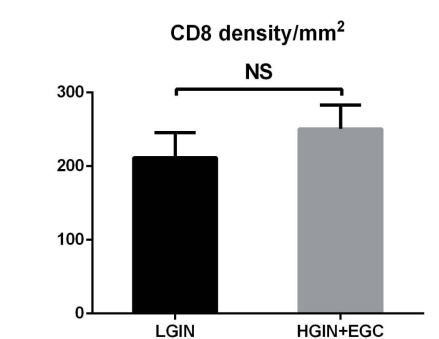

C

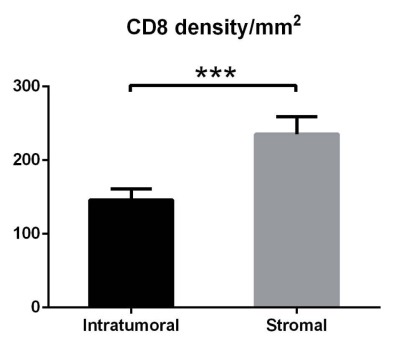

E
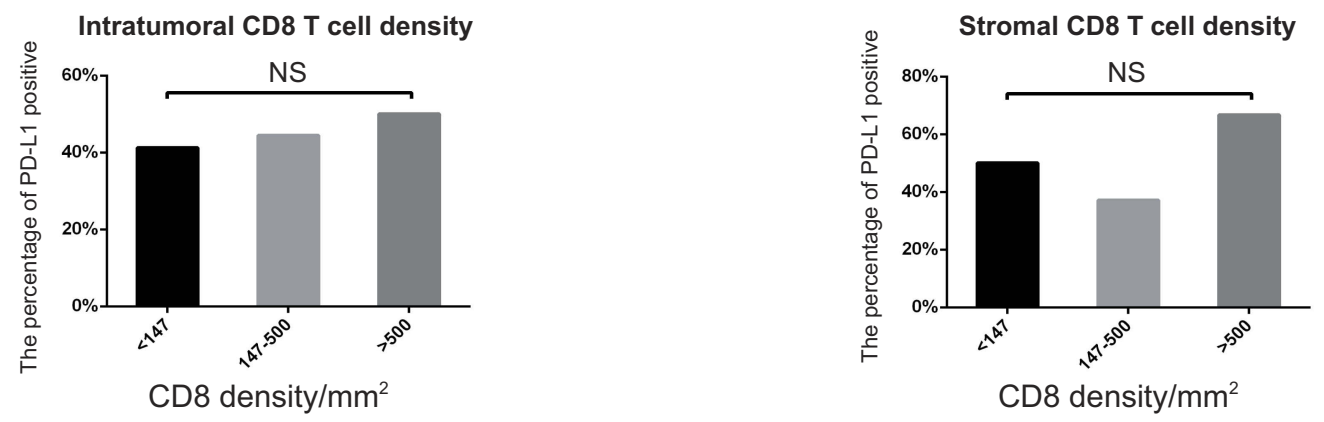

Figure 5 The CD8 density and its association with PD-LI expression in LGIN, HGIN, and EGC. (A) The representative intratumoral, tumor-stromal interface expression of CD8 in LGIN, HGIN, and EGC; (B) The correlation between stage and CD8 density; (C) CD8 density is more in tumor-stromal interface than in tumor (P=0.0005); (D, E) PD-LI is not associated with increasing CD8 density whatever in tumor-stromal interface and in tumor; CD8 density within LGIN, HGIN, and EGC was determined and densities were divided by quartiles into low $\left(<147 / \mathrm{mm}^{2}\right)$, mid $\left(147-500 / \mathrm{mm}^{2}\right)$, and high $\left(>500 / \mathrm{mm}^{2}\right)$. Correlation between CD8 density and PD-LI expression by location were determined using the exact version of the Cochran-Armitage trend test.

Note: $* * * * 0.001$.

Abbreviations: EGC, early-stage gastric cancer; HGIN, High-grade Intraepithelial neoplasia; LGIN, Low-grade Intraepithelial neoplasia; NS, not statistically significant; PDLI, programmed death-ligand I.

suggesting that an adaptive immune resistance-type mechanism is not active in intraepithelial neoplasia and EGC.

Although PD-L1 is expressed in pancreatic cancer cells, pancreatic cancer patients with high PD-L1 expression may not be responsive to anti-PD-L1 therapy. There are several reasons why anti-PD-L1 therapy is not ideal. First, the majority of pancreatic cancers are considered to be immuno-quiescent. Second, strong cancer antigens or epitopes recognized by $\mathrm{T}$-cells are scarce, resulting in minimal activation of cancer-specific T-cells. Third, infiltration of T-cells in pancreatic cancer tumor tissue is poor. ${ }^{25}$ The immune response in pancreatic cancer is similar to those seen in gastric intraepithelial neoplasia and EGC in our study. There are insufficient strong cancer antigens or epitopes recognized by T-cells in these early stages of gastric cancer, and the infiltration rate of $\mathrm{CD} 8+$ T-cells is relatively low, suggesting that poor infiltration and minimal activation of cancer-specific T-cells occurs. These results suggest that, despite positive expression of PD-L1 in gastric intraepithelial neoplasia and EGC, the therapeutic effect of anti-PD-L1 antibody therapy may be poor.

In advanced gastric cancer, upregulated PD-L1 on tumor cells interacts with PD-1 on activated T-cells, thus 
A

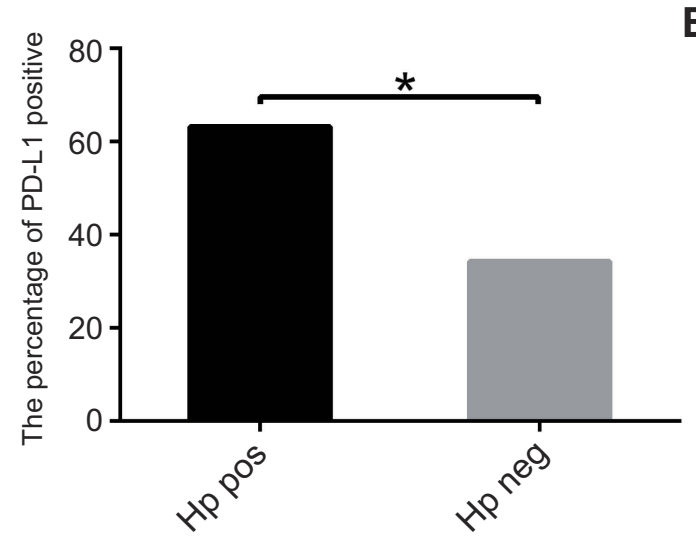

B

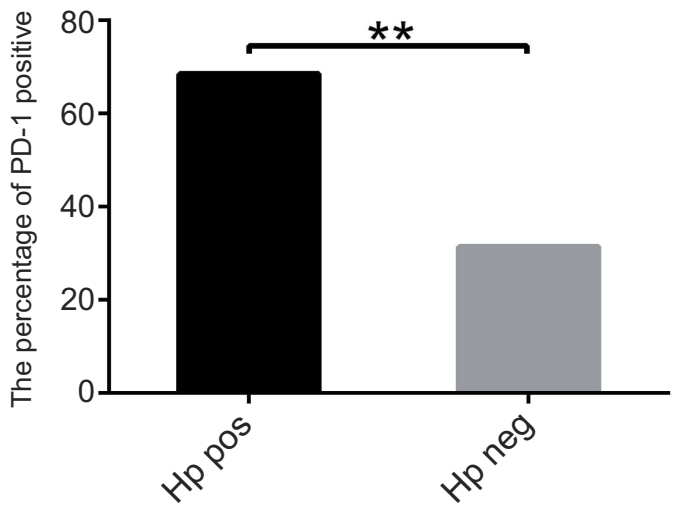

C

Normal gastic epithelium

Intraepithelial neoplasia

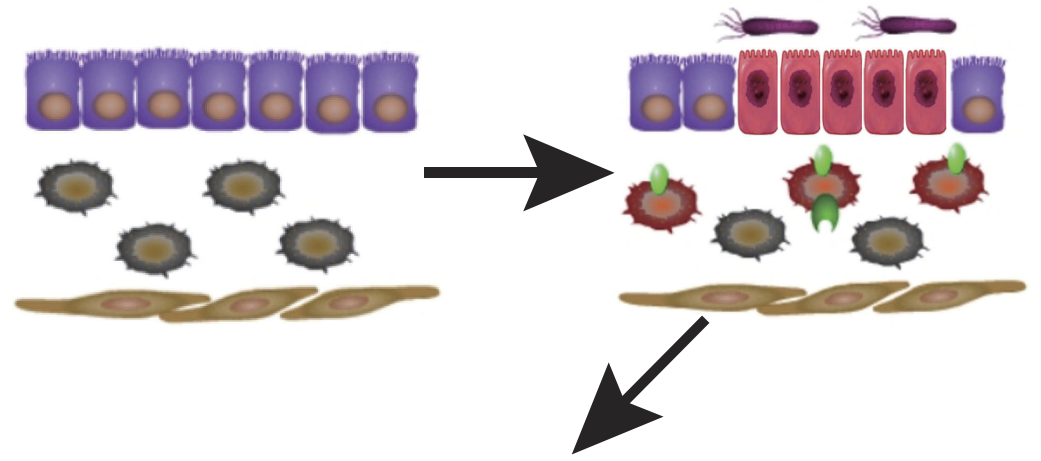

Gastric cancer
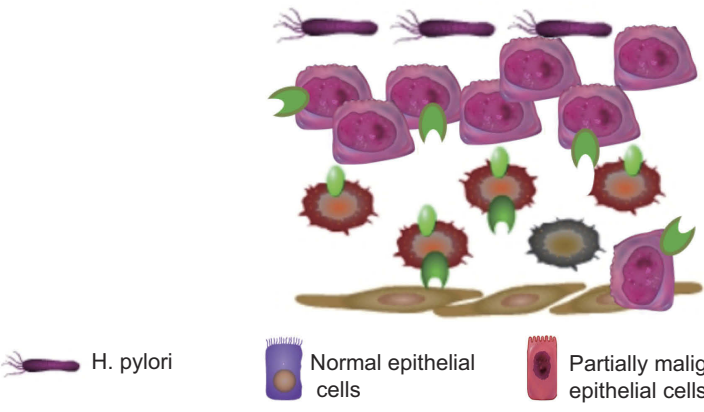

Partially malignant epithelial cells

Exhausted T cell Active T cell

PD-1

Tumor cell

A PD-L1

Figure 6 The relationship between Hp infection and PD-I, PD-LI expression. (A) The percentage of PD-LI positive in patients with HP positive and negative; (B) The percentage of PD-I positive in patients with Hp positive and negative. (C) Pattern diagram: Under chronic infection caused by Hp, the immune system may cause excessive damage of normal tissues. In order to avoiding excessive damage, PD-LI/PD-I checkpoint inhibition is upregulated, but also reduces the tumor killing effect of T-cells and promotes the progress of gastric cancer.

Note: $* P<0.05$ and $* * P<0.01$.

Abbreviations: Hp, Helicobacter pylori; PD-I, programmed death-I; PD-LI, programmed death-ligand I.

inhibiting T-cell function. However, PD-L1 expression was mainly detected on immune cells in our study, rather than on the tumor cells. We speculate that upregulation of PD-L1 on tumor cells is not the trigger for PD-L1/PD1-mediated immune inhibition, which may be induced by other factors. Under normal conditions, the PD-1/PD-L1 signal is an important inhibitory pathway and is balanced with costimulatory signals. ${ }^{7}$ Although PD-L1/PD-1 upregulation is necessary to maintain immune tolerance and prevention of excessive tissue damage during chronic infection, it may also cause tumor progression and immune escape. For example, PD-L1 expression was higher in patients with HPV infection, and PD-1 expression was similar to PD-L1 in intraepithelial neoplasia of cervical cancer. In particular, PD-1 was scarcely expressed in patients without HPV infection. ${ }^{15}$ 
Therefore, chronic infection may induce PD-L1/PD-1-mediated immune inhibition, thus limiting T-cell killing of tumor cells. Also, Hp colonization of the stomach can lead to chronic gastritis. ${ }^{16}$ We suggest that Hp may induce PD-1/PD-L1 expression and, thus, limit CD8+ T-cell activity, which is consistent with our findings that $\mathrm{Hp}$ infection correlates with PD-L1 expression. PD-L1 expression in $\mathrm{Hp}$ positive patients is greater than in $\mathrm{Hp}$ negative patients, therefore. PD-L1/PD-1-mediated inhibition is more active in patients with $\mathrm{Hp}$ infection.

It is well known that there are two mechanisms by which $\mathrm{Hp}$ infection promotes cancer. One mechanism involves the enhanced production of free radicals at the site of infection and an increased rate of host cell mutation. The other proposed mechanism is enhancement of the transformed host cell phenotype by means of alterations in cell proteins, such as adhesion proteins. ${ }^{26}$ In addition to the two mechanisms above, we speculate that there is another mechanism that may promote cancer progression in intraepithelial neoplasia and EGC. During chronic infection with $\mathrm{Hp}$, the immune system may cause excessive damage to normal tissues. To avoid such trauma, the PD-L1/PD-1 checkpoint is activated and upregulated, but this also reduces T-cell-mediated cytotoxicity and promotes the progression of gastric cancer. In our study, Hp infection was positively correlated with PD-L1 expression and higher grade of lesion, consistent with our speculation. This ultimately allows tumors to develop following escape from immune surveillance.

As summarized in Figure 6C, we compared the expression of PD-L1 and PD-1 in LGIN, HGIN, and EGC, and the association with CD8+ T-cells and Hp infection. Our results suggest that the $\mathrm{PD}-1 / \mathrm{PD}-\mathrm{L} 1$ inhibitory axis may be activated in LGIN, HGIN, and EGC, and this was associated with Hp infection. This may be an important starting factor for induction of checkpoint-mediated immune inhibition which further limits the tumoricidal effect of T-cells and promotes the progression of gastric cancer.

\section{Abbreviation list}

PD-L1, Programmed death-ligand 1; PD-1, Programmed death-1; LGIN, Low-grade Intraepithelial neoplasia; HGIN, High-grade Intraepithelial neoplasia; EGC, earlystage gastric cancer; FACS, Flow cytometric analysis; PBMC, peripheral blood mononuclear cell; Hp, Helicobacter pylori; LCI, Linked Color Imaging; BLI, Blue laser imaging; CIN, Cervical Intraepithelial Neoplasia.

\section{Ethical approval}

All procedures performed in studies involving human participants were in accordance with the ethical standards of the institutional and/or national research committee (Institutional Review Board of Ruijin Hospital, Shanghai, China) and with the 1964 Declaration of Helsinki and its later amendments

\section{Acknowledgments}

We thank all members of the Lab of Subing who helped with the flow cytometry analysis of PD-1 on CD4+ T-cell and CD8+ T-cell in the LGIN, HGIN, and EGC. We also thank Gillian Campbell, PhD, from Liwen Bianji, Edanz Group China (www.liwenbianji.cn/ac), for editing the English text of a draft of this manuscript.

\section{Disclosure}

The authors report no conflicts of interest in this work.

\section{References}

1. Ferlay J, Steliarova-Foucher E, Lortet-Tieulent J, et al. Cancer incidence and mortality patterns in Europe: estimates for 40 countries in 2012. Eur $J$ Cancer. 2013;49(6):1374-1403. doi:10.1016/j. ejca.2012.12.027

2. Muro K, Chung HC, Shankaran V, et al. Pembrolizumab for patients with PD-L1-positive advanced gastric cancer (KEYNOTE-012): a multicentre, open-label, phase 1b trial. Lancet Oncol. 2016;17 (6):717-726. doi:10.1016/S1470-2045(16)00175-3

3. Le DT, Durham JN. Mismatch repair deficiency predicts response of solid tumors to PD-1 blockade. Science. 2017;357(6349):409-413. doi:10.1126/science.aan6733

4. Le DT, Uram JN, Wang H, et al. PD-1 blockade in tumors with mismatch-repair deficiency. $N$ Engl $J$ Med. 2015;372 (26):2509-2520. doi:10.1056/NEJMoa1500596

5. Tumeh PC, Harview CL, Yearley JH, et al. PD-1 blockade induces responses by inhibiting adaptive immune resistance. Nature. 2014;515(7528):568-571. doi:10.1038/nature 13954

6. Pardoll DM. The blockade of immune checkpoints in cancer immunotherapy. Nat Rev Cancer. 2012;12(4):252-264. doi:10.1038/ $\operatorname{nrc} 3239$

7. Silva R, Gullo I, Carneiro F. The PD-1: PD-L1immune inhibitory checkpoint in Helicobacter pylori infection and gastric cancer: a comprehensive review and future perspectives. Porto Biomed J. 2016;1(1):4-11. doi:10.1016/j.pbj.2016.03.004

8. Fuchs CS, Doi T, Jang RW-J, et al. KEYNOTE-059 cohort 1: efficacy and safety of pembrolizumab (pembro) monotherapy in patients with previously treated advanced gastric cancer. J Clin Oncol. 2017;35 (15_suppl):4003. doi:10.1200/JCO.2017.35.15_suppl.4003

9. Thompson ED, Zahurak M, Murphy A, et al. Patterns of PD-L1 expression and CD8 $\mathrm{T}$ cell infiltration in gastric adenocarcinomas and associated immune stroma. Gut. 2017;66(5):794-801. doi:10.1136/gutjnl-2015-310839

10. Xing X, Guo J, Wen X, et al. Analysis of PD1, PDL1, PDL2 expression and $\mathrm{T}$ cells infiltration in 1014 gastric cancer patients. Oncoimmunology. 2018;7(3):e1356144. doi:10.1080/ 2162402X.2018.1490854

11. Tamura T, Ohira M, Tanaka H, et al. Programmed death-1 ligand-1 (PDL1) expression is associated with the prognosis of patients with stage II/III gastric cancer. Anticancer Res. 2015;35(10):5369-5376. 
12. Spranger S, Spaapen RM, Zha Y, et al. Up-regulation of PD-L1, IDO, and Tregs in the melanoma tumor microenvironment is driven by CD8+ T cells. Sci Transl Med. 2013;5(200):200ra116. doi:10.1126/ scitranslmed.3006504

13. Acosta-Gonzalez G, Ouseph M, Lombardo K, Lu S, Glickman J, Resnick M. Immune environment in serrated lesions of the colon: intraepithelial lymphocyte density, PD-1, and PD-L1 expression correlate with serrated neoplasia pathway progression. Hum Pathol. 2018;83:115-123. doi:10.1016/j.humpath.2018.08.020

14. Mezache L, Paniccia B, Nyinawabera A, Nuovo GJ. Enhanced expression of PD L1 in cervical intraepithelial neoplasia and cervical cancers. Mod Pathol. 2015;28(12):1594-1602. doi:10.1038/ modpathol.2015.108

15. Yang W, Song Y, Lu YL, Sun JZ, Wang HW. Increased expression of programmed death (PD)-1 and its ligand PD-L1 correlates with impaired cell-mediated immunity in high-risk human papillomavirus-related cervical intraepithelial neoplasia. Immunology. 2013;139(4):513-522. doi:10.1111/imm.12101

16. Wroblewski LE, Peek RM Jr., Wilson KT. Helicobacter pylori and gastric cancer: factors that modulate disease risk. Clin Microbiol Rev. 2010;23(4):713-739. doi:10.1128/CMR.00011-10

17. Mommersteeg MC, Yu J, Peppelenbosch MP, Fuhler GM. Genetic host factors in Helicobacter pylori-induced carcinogenesis: emerging new paradigms. Biochimica Et Biophysica Acta Rev Cancer. 2018;1869(1):42-52. doi:10.1016/j.bbcan.2017.11.003

18. Rugge M, Genta RM, Di Mario F, et al. Gastric cancer as preventable disease. Clin Gastroenterol Hepatol. 2017;15(12):1833-1843. doi:10.1016/j.cgh.2017.05.023
19. Malfertheiner P, Megraud F, O`Morain CA, et al. Management of Helicobacter pylori infection-the Maastricht V/Florence consensus report. Gut. 2017;66(1):6-30. doi:10.1136/gutjnl-2016312288

20. Das S, Suarez G, Beswick EJ, Sierra JC, Graham DY, Reyes VE. Expression of B7-H1 on gastric epithelial cells: its potential role in regulating $\mathrm{T}$ cells during Helicobacter pylori infection. J Immunol. 2006;176(5):3000-3009. doi:10.4049/jimmunol.176.5.3000

21. Wu YY, Chen JH, Kao JT, et al. Expression of CD25(high) regulatory $\mathrm{T}$ cells and PD-1 in gastric infiltrating CD4(+) T lymphocytes in patients with Helicobacter pylori infection. Clin Vaccine Immunol. 2011;18(7):1198-1201. doi:10.1128/CVI.00422-10

22. Topalian SL, Hodi FS, Brahmer JR, et al. Safety, activity, and immune correlates of anti-PD-1 antibody in cancer. $N$ Engl J Med. 2012;366(26):2443-2454. doi:10.1056/NEJMoa1200690

23. Velcheti V, Schalper KA, Carvajal DE, et al. Programmed death ligand-1 expression in non-small cell lung cancer. Lab Invest. 2014;94(1):107-116. doi:10.1038/labinvest.2013.130

24. Herbst RS, Soria JC, Kowanetz M, et al. Predictive correlates of response to the anti-PD-L1 antibody MPDL3280A in cancer patients. Nature. 2014;515(7528):563-567. doi:10.1038/nature14011

25. Feng M, Xiong G, Cao Z, et al. PD-1/PD-L1 and immunotherapy for pancreatic cancer. Cancer Lett. 2017;407:57-65. doi:10.1016/j. canlet.2017.08.006

26. Tsuji S, Kawai N, Tsujii M, Kawano S, Hori M. Review article: inflammation-related promotion of gastrointestinal carcinogenesis-a perigenetic pathway. Aliment Pharmacol Ther. 2003;18(Suppl $1): 82-89$.
Cancer Management and Research

\section{Publish your work in this journal}

Cancer Management and Research is an international, peer-reviewed open access journal focusing on cancer research and the optimal use of preventative and integrated treatment interventions to achieve improved outcomes, enhanced survival and quality of life for the cancer patient.
The manuscript management system is completely online and includes a very quick and fair peer-review system, which is all easy to use. Visit http://www.dovepress.com/testimonials.php to read real quotes from published authors. 